\title{
Optimal Contrast Concentration for CT-Guided Epidural Steroid Injections
}

\author{
P.G. Kranz, M. Abbott, D. Abbott, and J.K. Hoang
}

\begin{abstract}
BACKGROUND AND PURPOSE: Contrast is used in CT-guided epidural steroid injections to ensure proper needle placement. Once injected, undiluted contrast often obscures the needle, hindering subsequent repositioning. The purpose of this investigation was to establish the optimal contrast dilution for CT-guided epidural steroid injections.
\end{abstract}

MATERIALS AND METHODS: This investigation consisted of an initial phantom study, followed by a prospective, randomized, singlecenter trial assessing a range of contrast dilutions. In the phantom study, a phantom housing a chamber containing a 22-gauge needle and various dilutions of contrast was scanned, and images were evaluated for needle visibility. On the basis of these results, concentrations of $66,100,133$, and $150 \mathrm{mg} / \mathrm{mL}$ iodine were selected for evaluation in a clinical study. Patients presenting for CT-guided epidural steroid injections were randomly assigned to a contrast dilution, and images from the procedure were evaluated by 2 readers blinded to the contrast assignment. Needle visibility was scored by use of a 5-point scale.

RESULTS: In the phantom study, the needle was not visible at contrast concentrations of $\geq 133 \mathrm{mg} / \mathrm{mL}$. In the clinical study, needle visibility was strongly associated with contrast concentration $(P<.0001)$. Significant improvements in visibility were found in $66 \mathrm{mg} / \mathrm{mL}$ and $100 \mathrm{mg} / \mathrm{mL}$ compared with higher iodine concentrations; no difference was found comparing $66 \mathrm{mg} / \mathrm{mL}$ with $100 \mathrm{mg} / \mathrm{mL}$ iodine. Neither injection location (cervical versus lumbar) nor technique (interlaminar versus transforaminal) influenced visibility scores.

CONCLUSIONS: For CT-guided epidural steroid injections, the optimal contrast concentration is $66-100 \mathrm{mg} / \mathrm{mL}$ iodine. Because these concentrations are not commercially available, proceduralists must dilute their contrast for such procedures.

ABBREVIATIONS: ESI = epidural steroid injections; ILESI = interlaminar epidural steroid injection; TFESI = transforaminal epidural steroid injection; $C$ TF $=C$ C fluoroscopy

maging guidance is a critical component of safe and accurate needle placement during spinal epidural steroid injections (ESI). ${ }^{1-6}$ The injection of contrast medium during such imageguided procedures provides information on patterns of injectate spread and helps to avoid complications caused by inadvertent intrathecal or intravascular needle placement. When the pattern of injected contrast shows that the needle position is not ideal, the proceduralist may reposition the needle.

Although conventional fluoroscopy remains the most widely used technique for guidance, CT fluoroscopy (CTF) has become

Received January 4, 2013; accepted after revision March 20.

From the Departments of Radiology (P.G.K., M.A., J.K.H.) and Biostatistics and Bioinformatics (D.A.), Duke University Medical Center, Durham, North Carolina.

Paper previously presented in part at: Annual Meeting of the American Society of Neuroradiology, April 26, 2012; New York, New York.

Please address correspondence to Peter G. Kranz, MD, Department of Radiology, Box 3808, Duke University Medical Center, Durham, NC 27710; e-mail: peter.kranz@duke.edu

Evidence-Based Medicine Level 1.

http://dx.doi.org/10.3174/ajnr.A3626 increasingly used because of its ability to provide cross-sectional needle localization and excellent visualization of the regional soft tissues. ${ }^{7-10}$ When CT guidance is used, however, the attenuation of many commercially available contrast agents is often sufficient to obscure the needle tip after injection. This can hinder attempts at needle repositioning, and as a result, several authors have advocated the use of dilute contrast for CT-guided procedures. The recommendations for the degree of contrast dilution vary widely, have generally been empirically selected, and, to our knowledge, have not been systematically evaluated previously.

The purpose of this investigation was to establish the optimal iodine concentration in contrast material for use in CT-guided spine procedures. Our study design included a phantom study for the purpose of acquiring preliminary data, followed by a prospective, randomized trial of various contrast dilutions in conditions mirroring routine clinical practice.

\section{MATERIALS AND METHODS}

This investigation was conducted in 2 parts. The first part was a phantom study designed to narrow the range of contrast dilutions 

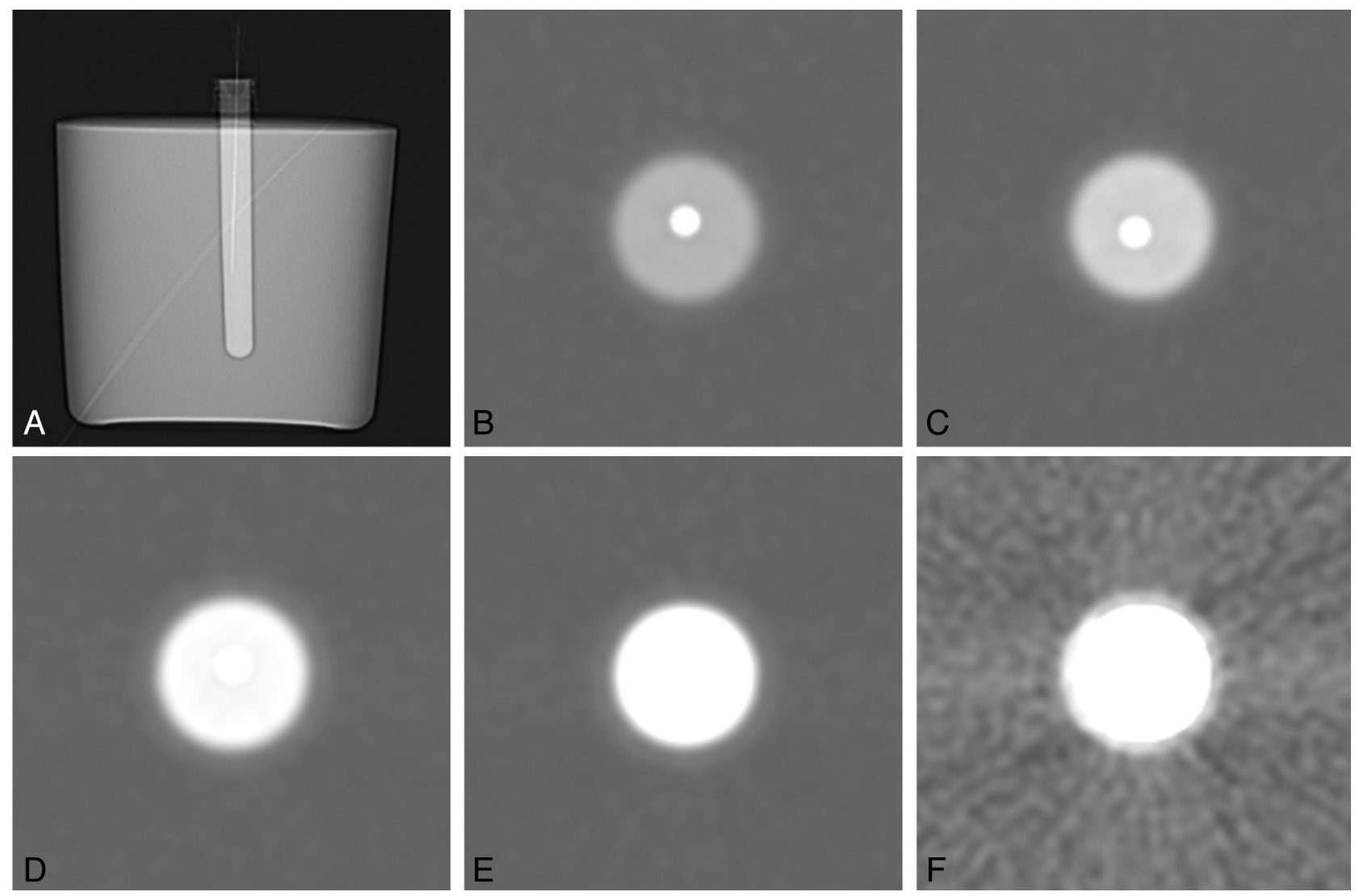

FIG 1. Phantom study assessing needle visibility. A, Scout image from CT demonstrating the configuration of the phantom. The central chamber houses contrast and a 22-gauge needle. Axial cross-sectional images of the chamber are shown with various contrast dilutions: $50 \mathrm{mg} / \mathrm{mL}(B), 66$ $\mathrm{mg} / \mathrm{mL}$ (C), $100 \mathrm{mg} / \mathrm{mL}(D), 133 \mathrm{mg} / \mathrm{mL}$ (E), and $150 \mathrm{mg} / \mathrm{mL}$ (F).

to be tested. The second part was a prospective, randomized, single-center trial assessing a range of contrast dilutions in actual CTF-guided epidural steroid injections. The study was approved by our local institutional review board and was compliant with the Health Insurance Portability and Accountability Act.

\section{Part I: Phantom Study}

We constructed a 15-cm-diameter cylindrical phantom composed of ballistics-grade gelatin ( $12 \% \mathrm{wt} / \mathrm{vol})$ that housed a central cylindrical chamber capable of holding contrast. Contrast dilutions were prepared by diluting commercially available contrast medium containing $200 \mathrm{mg} / \mathrm{mL}$ iodine (iopamidol; Isovue-M 200, Bracco Diagnostics, Princeton, New Jersey) with preservative-free sterile saline to achieve 5 final iodine concentrations: 50 , $66,100,133$, and $150 \mathrm{mg} / \mathrm{mL}$. The phantom's central chamber was filled with various dilutions of contrast, and a 22-gauge needle was secured into place, centered along the long axis of the chamber. The phantom was placed in a clinical CT scanner (LightSpeed 16; GE Healthcare, Milwaukee, Wisconsin) containing each contrast dilution and was scanned by use of the axial technique at a tube current of $10 \mathrm{~mA}$ and peak voltage of $120 \mathrm{kVp}$ (Fig 1). The images were then reviewed by a neuroradiologist by use of the scanner's preset window/level setting for CTF procedures (window and level setting: 4000/465). Needle visibility was assessed by use of a 3-point scoring system, with a score of 0 indicating clear visibility of the needle against the background contrast, a score of 1 indicating borderline visibility, necessitating adjustment of the window and level settings, and a score of 2 indicating inability to visualize the needle.

\section{Part II: Clinical Study}

On the basis of the results of the phantom study, we selected 4 contrast concentrations for use in the clinical study: 66, 100, 133, and $150 \mathrm{mg} / \mathrm{mL}$ iodine. Subjects were recruited from among adult patients presenting to a single neuroradiologist for CTF-guided interlaminar or transforaminal epidural steroid injections in the cervical or lumbar spine. A contraindication to iodinated contrast, such as a prior allergic reaction, was an exclusion criterion.

Once enrolled, subjects were randomly assigned to one of the contrast dilutions on the basis of a predetermined randomization schedule, and the clinically indicated procedure was performed. For patients scheduled for multiple injections in a single visit, a single-contrast dilution was used for all injections performed at that visit. All scans were performed on the same clinical scanner, which was the same scanner used to scan the phantom. For each procedure, tube current settings were at the discretion of the proceduralist and were based on body habitus and injection location.

\section{Image Analysis}

Images from the procedures were reviewed by use of a standardized window and level setting $(4000 / 465)$ by 2 neuroradiologists, each with 5 years of experience performing CTF- guided steroid injections. The readers were blinded to the assigned contrast dilution. One of the readers was the same radiologist who per- 
formed the injections; however, image review occurred 5 months after the end of patient enrollment, thereby mitigating the chance that any particular dilution could be recalled during analysis. Images were scored according to a 5-point scoring system (Table 1), which expanded the 3-point system used in the phantom study. Scores of -2 and -1 were added to account for the need to achieve adequate anatomic soft tissue contrast when performing clinical procedures. Representative examples of injections scored as optimal and at the extremes of the 5-point scale are shown in Fig 2 . In 4 cases in which the readers disagreed by 2 points, the case was jointly reviewed by both readers and a consensus score was determined. Injection technique (interlaminar epidural steroid injection [ILESI] versus transforaminal epidural steroid injection [TFESI]) was also recorded.

\section{Data Analysis}

Data were analyzed with the use of SAS software (version 9.3, SAS Institute, Cary, North Carolina). Because there was more than 1 injection per subject in some cases, visibility scores were analyzed by means of the generalized estimating equations test of difference, assuming a multinomial distribution and a cumulative logit link function. A value of $P=.05$ was considered to be statistically significant for analysis of the effect of contrast concentration on needle visibility. Follow-up contrast statements in the generalized estimating equations test of difference model comparing multiple individual contrast concentrations as well as the effect of injection type (ie, ILESI versus TFESI) were analyzed with the use of a Bonferroni-corrected significance threshold of 0.0083 .

\section{RESULTS}

In the phantom study, iodine concentrations of 50 and $66 \mathrm{mg} / \mathrm{mL}$ were associated with clear needle visualization, $100 \mathrm{mg} / \mathrm{mL}$ was found to be borderline for needle visualization, and concentra-

Table 1: Five-point scoring system for the clinical study

\begin{tabular}{lcllll}
\hline & Too Concentrated & & Optimal & Too Dilute \\
\hline Visibility score & +2 & +1 & 0 & -1 & -2 \\
Needle tip & Not visible & Borderline & Visible & Visible & Visible \\
Soft tissue contrast & Adequate & Adequate & Adequate & Borderline & Not acceptable \\
\hline
\end{tabular}
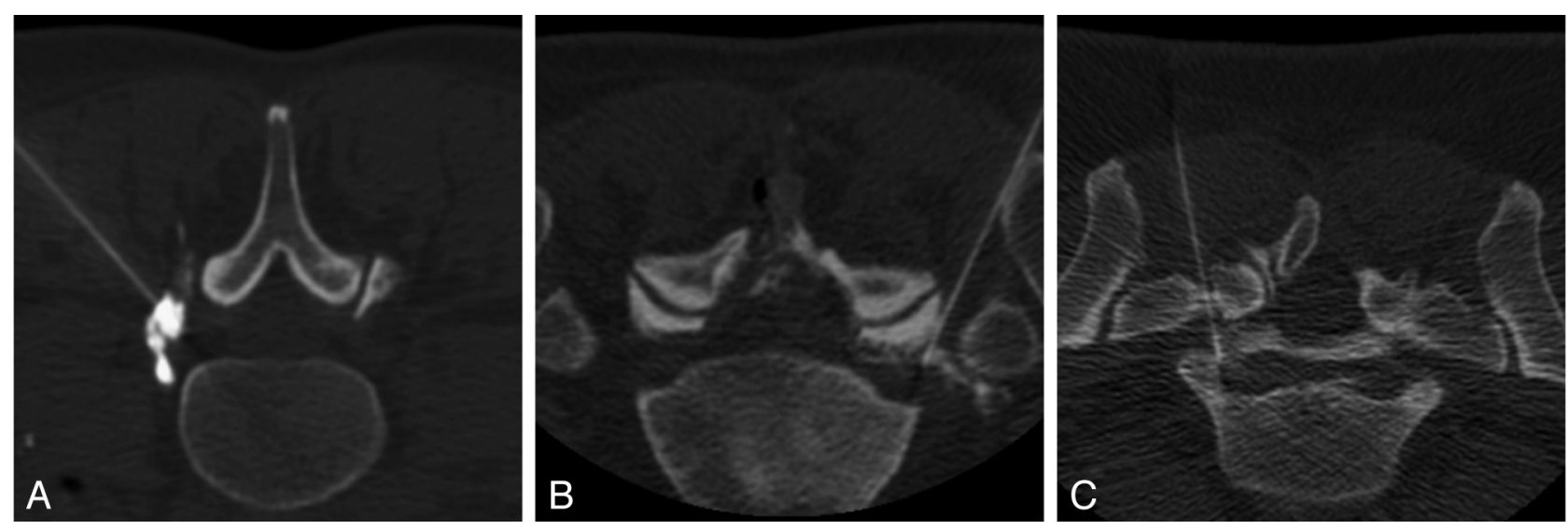

FIG 2. Axial CTF images show representative examples of visibility scores on the basis of our 5 -point scoring system. $A$, Score of +2 . The contrast is too dense to visualize the location of the needle tip. $B$, Score of 0 . The needle tip can be distinguished from the injected contrast; adequate soft tissue contrast is also provided. C, Score of -2 . The injected contrast is too dilute to provide adequate soft tissue contrast. 
Table 2: Visibility scores for the clinical study

\begin{tabular}{cccccc}
\hline & \multicolumn{5}{c}{ Visibility Score } \\
\cline { 2 - 6 } & $-\mathbf{- 2}$ & $\mathbf{- 1}$ & $\mathbf{0}$ & $\mathbf{+ 1}$ & $\mathbf{+ 2}$ \\
\hline Total scores $(n=120)$ & $n=9$ & $n=10$ & $n=34$ & $n=25$ & $n=42$ \\
(2 readers) & $(7.5 \%)$ & $(8.3 \%)$ & $(28.3 \%)$ & $(20.8 \%)$ & $(35.0 \%)$ \\
lodine concentration & & & & & \\
$\quad 66 \mathrm{mg} / \mathrm{mL}(n=34)$ & $n=7$ & $n=5$ & $n=15$ & $n=6$ & $n=1$ \\
& $(20.6 \%)$ & $(14.7 \%)$ & $(44.1 \%)$ & $(17.6 \%)$ & $(2.9 \%)$ \\
$100 \mathrm{mg} / \mathrm{mL}(n=32)$ & $n=2$ & $n=5$ & $n=9$ & $n=10$ & $n=6$ \\
& $(6.3 \%)$ & $(15.6 \%)$ & $(28.1 \%)$ & $(31.3 \%)$ & $(18.8 \%)$ \\
$133 \mathrm{mg} / \mathrm{mL}(n=26)$ & $n=0$ & $n=0$ & $n=2$ & $n=3$ & $n=21$ \\
& $(0 \%)$ & $(0 \%)$ & $(7.7 \%)$ & $(11.5 \%)$ & $(80.8 \%)$ \\
$150 \mathrm{mg} / \mathrm{mL}(n=28)$ & $n=0$ & $n=0$ & $n=8$ & $n=6$ & $n=14$ \\
& $(0 \%)$ & $(0 \%)$ & $(28.6 \%)$ & $(21.4 \%)$ & $(50.0 \%)$ \\
Injection technique & & & & & \\
ILESI $(n=64)$ & $n=0$ & $n=3$ & $n=14$ & $n=17$ & $n=30$ \\
& $(0 \%)$ & $(4.7 \%)$ & $(21.9 \%)$ & $(26.6 \%)$ & $(46.9 \%)$ \\
TFESI $(n=56)$ & $n=9$ & $n=7$ & $n=20$ & $n=8$ & $n=12$ \\
& $(16.1 \%)$ & $(12.5 \%)$ & $(35.7 \%)$ & $(14.3 \%)$ & $(21.4 \%)$ \\
\hline
\end{tabular}

To our knowledge, ours is the first investigation to systematically evaluate the effect of contrast concentration on needle visibility in CT-guided procedures. Previous authors have recommended the use of dilute contrast for use in CT-guided procedures, but the suggested dilutions have generally been empirically determined and vary widely among authors. For cervical TFESI, contrast dilutions of $1: 10,1: 2,1: 1$, and $3: 1$ have been reported by different authors, with the use of either saline or local anesthetic as the diluent. ${ }^{7,10-13}$ A report of CT-guided celiac plexus neurolysis technique recommended the use of a contrast dilution of 1:4 with anesthetic. ${ }^{14}$ In many cases, the dilution ratios were re-

Table 3: Comparison of visibility scores on the basis of combined iodine concentration and injection technique

\begin{tabular}{lc}
\hline \multicolumn{1}{c}{ lodine Concentration + Injection Technique } & $\boldsymbol{P}$ Value $^{\mathrm{a}}$ \\
\hline$(66 \mathrm{mg} / \mathrm{mL}$ and TFESI) versus (100 mg/mL and TFESI) & .011 \\
$(66 \mathrm{mg} / \mathrm{mL}$ and ILESI) versus $(100 \mathrm{mg} / \mathrm{mL}$ and ILESI) & .016 \\
$(66 \mathrm{mg} / \mathrm{mL}$ and ILESI) versus $(66 \mathrm{mg} / \mathrm{mL}$ and TFESI) & .051 \\
$(100 \mathrm{mg} / \mathrm{mL}$ and ILESI) versus (100 mg/mL and TFESI) & .021 \\
\hline
\end{tabular}

a $P$ values are based on results of generalized estimating equations test of difference contrast statements with the use of a Bonferroni-corrected significance threshold of 0.0083 .

To determine whether either the $66 \mathrm{mg} / \mathrm{mL}$ or $100 \mathrm{mg} / \mathrm{mL}$ concentration was superior for a particular injection technique (ILESI versus TFESI), we examined multiple combinations of contrast concentration and injection technique. These included comparison of visibility scores for TFESI with scores for ILESI at both $66 \mathrm{mg} / \mathrm{mL}$ and $100 \mathrm{mg} / \mathrm{mL}$ iodine, as well as scores for TFESI injections performed at $66 \mathrm{mg} / \mathrm{mL}$ with those performed at $100 \mathrm{mg} / \mathrm{mL}$, and ILESI scores for the same concentrations (Table 3 ). Although we observed trends for several of these combinations, none of these comparisons achieved statistical significance by use of the corrected significance threshold of 0.0083 .

\section{DISCUSSION}

The role of contrast in ESI is to confirm the epidural and extravascular location of the needle tip and to examine the spread of injectate toward the therapeutic target. In cases in which the contrast pattern indicates an undesirable needle position necessitating repositioning of the needle, the contrast attenuation must not obscure the visibility of the needle tip. Our investigation demonstrated that iodine concentration was highly associated with needle visibility $(P<.0001)$ during CT-guided ESI and that at or above iodine concentrations of $133 \mathrm{mg} / \mathrm{mL}$, the contrast was too attenuated to allow needle visualization. Furthermore, our results suggest that the optimal iodine concentration for such procedures is $66-100 \mathrm{mg} / \mathrm{mL}$. Because commercially available contrast media used for spinal injections generally are available with iodine concentration of $180-200 \mathrm{mg} / \mathrm{mL}$ and higher, proceduralists seeking to optimize needle visibility during CT-guided spine procedures must dilute their contrast. ported, but details of the contrast medium being diluted or its iodine concentration were not specified. Thus, our study aimed to systematically determine the optimal iodine concentration for use in CT-guided ESI. In our investigation, the contrast concentrations that produced the highest proportions of acceptable scores correspond to dilutions of 1:1 (100 mg/mL) or 1:2 (66 mg/mL).

Visibility is particularly important when performing CTguided procedures requiring high degrees of precision in needle placement, such as with cervical epidural steroid injections (Fig 3). In a previous investigation, we found that needle repositioning was necessary after contrast injection in $16 \%$ of cervical TFESIs because of the undesirable spread of contrast in the epidural space, subperiosteal injection, or intravascular injection. ${ }^{15}$ In cases in which critical neural and vascular structures are in close proximity to the target needle placement, the inability to visualize the needle tip and make controlled adjustments to its position increases the risk for catastrophic consequences. The need to decrease iodine concentration to allow visibility of the needle must be balanced against the need for adequate soft tissue contrast. Overdilution of contrast could result in inability to confidently confirm epidural location of the needle, failure to adequately assess the extent and direction of spread of injectate in the epidural space, or failure to detect intrathecal or intravascular injection. The ideal concentration of contrast would permit needle visibility while maintaining good visibility of the contrast as it disperses in the soft tissues surrounding the injection site.

The 5-point scoring system that we used for the clinical study was designed to balance these competing needs. We found that needle visibility against the background contrast pool is no longer possible at concentrations $\geq 133 \mathrm{mg} / \mathrm{mL}$ under phantom conditions and is unacceptable in most clinical cases. Notably, we did not find a statistically significant difference in visibility scores between the $66 \mathrm{mg} / \mathrm{mL}$ and $100 \mathrm{mg} / \mathrm{mL}$ concentrations, nor did we identify a subgroup on the basis of injection location or technique in which one concentration or the other was superior. We did find that at a concentration of $100 \mathrm{mg} / \mathrm{mL}$ iodine, most unacceptable visibility scores resulted from a contrast concentration too high to allow needle visibility. Conversely, at $66 \mathrm{mg} / \mathrm{dL}$, most unacceptable scores resulted from overdiluted contrast and insufficient 

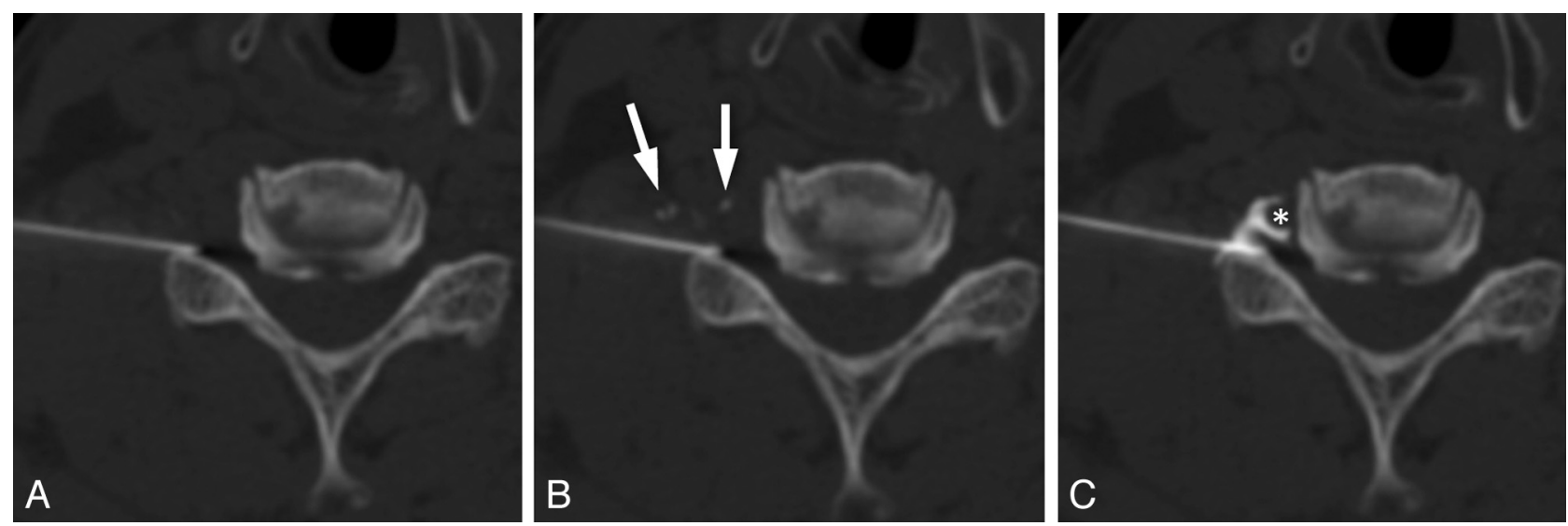

FIG 3. Importance of precise needle placement in cervical epidural steroid injections. A, Axial CTF image obtained before contrast injection. $B$, Image obtained immediately after contrast injection shows transient filling of small vessels (arrows). No epidural spread is seen. C, Image obtained after advancing the needle $<2 \mathrm{~mm}$ demonstrates foraminal epidural contrast spread, with no vascular filling. Note the close proximity of the needle tip to the vertebral artery $\left.{ }^{*}\right)$. This case highlights the importance of accurate localization of the needle tip and the need to be able to make small but precise adjustments in needle position during epidural injections.

soft tissue contrast. Yet, for both dilutions, there were cases scored as too concentrated (ie, score of +2 ) as well as others scored as too dilute (ie, score of -2), suggesting that some component of needle visibility may depend on idiosyncratic factors related to the anatomy or tissue composition of the injection site or individual perceptual differences by the scorers. Although our investigation focused on ESI, a variety of other CT-guided procedures use contrast injection, and we would anticipate that our results would be generalizable to other techniques in which contrast is injected into soft tissue.

There are several limitations to this investigation. First, the number of injections in this prospective investigation was selected to evaluate our primary hypothesis that there would be a difference in needle visibility on the basis of concentration. There are subgroups of injections, however, for which our total number of subjects may be insufficiently powered to detect differences. With larger numbers, it is possible that differences in optimal contrast concentration could be found between interlaminar epidural and transforaminal injections in which we observed nonsignificant trends or between cervical and lumbar injections. Second, the degree to which any single contrast attenuation is considered optimal may be in part influenced by the specific considerations of the procedure being performed, the relative hazards of overdilution versus underdilution of contrast in any particular context, and by the subjective preferences of the proceduralist. Our determination of optimal iodine concentration should be considered a general guide but can be tailored according to the needs of individual cases and operators.

\section{CONCLUSIONS}

Optimal iodine concentration in contrast used during CT-guided spine procedures is $66-100 \mathrm{mg} / \mathrm{mL}$. This range provides the greatest balance between visualizing the needle and providing adequate tissue contrast. Because these concentrations are not currently commercially available, the use of a 2:1 or 1:1 dilution of contrast by proceduralists performing CT-guided ESI would be necessary, assuming a starting contrast concentration of 200 $\mathrm{mg} / \mathrm{mL}$ iodine.

\section{REFERENCES}

1. Provenzano DA, Fanciullo G. Cervical transforaminal epidural steroid injections: should we be performing them? Reg Anesth Pain Med 2007;32:169-70

2. McGrath JM, Schaefer MP, Malkamaki DM. Incidence and characteristics of complications from epidural steroid injections. Pain Med 2011;12:726-31

3. Benny B, Azari P, Briones D. Complications of cervical transforaminal epidural steroid injections. Am $J$ Phys Med Rehabil 2010;89:601-07

4. White AH, Derby R, Wynne G. Epidural injections for the diagnosis and treatment of low-back pain. Spine 1980;5:78-86

5. Stojanovic MP, Vu TN, Caneris O, et al. The role of fluoroscopy in cervical epidural steroid injections: an analysis of contrast dispersal patterns. Spine 2002;27:509-14

6. Glaser SE, Shah RV. Root cause analysis of paraplegia following transforaminal epidural steroid injections: the 'unsafe' triangle. Pain Physician 2010;13:237-44

7. Wald JT, Maus TP, Geske JR, et al. Safety and efficacy of CT-guided transforaminal cervical epidural steroid injections using a posterior approach. AJNR Am J Neuroradiol 2012;33:415-19

8. Chang A, Pochert S, Romano C, et al. Safety of $\mathbf{1 0 0 0}$ CT-guided steroid injections with air used to localize the epidural space. AJNR Am J Neuroradiol 2011;32:E175-77

9. Silbergleit R, Mehta BA, Sanders WP, et al. Imaging-guided injection techniques with fluoroscopy and CT for spinal pain management. Radiographics 2001;21:927-39

10. Wagner AL. CT fluoroscopic-guided cervical nerve root blocks. AJNR Am J Neuroradiol 2005;26:43-44

11. Hoang JK, Apostol MA, Kranz PG, et al. CT fluoroscopy-assisted cervical transforaminal steroid injection: tips, traps, and use of contrast material. AJR Am J Roentgenol 2010;195:888-94

12. Miller TS, Fruauff K, Farinhas J, et al. Lateral decubitus positioning for cervical nerve root block using CT image guidance minimizes effective radiation dose and procedural time. AJNR Am J Neuroradiol 2013;34:23-28

13. Kranz PG, Raduazo PA. Technique for CT fluoroscopy-guided cervical interlaminar steroid injections. $A J R A m J$ Roentgenol 2012;198:675-77

14. Wang PJ, Shang MY, Qian Z, et al. CT-guided percutaneous neurolytic celiac plexus block technique. Abdom Imaging 2006;31:710-18

15. Hoang JK, Massoglia DP, Apostol MA, et al. CT-guided cervical transforaminal steroid injections: where should the needle tip be located? AJNR Am J Neuroradiol 2013;34:688-92 